\title{
LA RESTAURACIÓN ARQUITECTÓNICA EN GALICIA. REFLEXIONES
}

\author{
Por \\ CONCHA FONTENLA SAN JUAN
}

\section{OBJETIVOS}

El objetivo principal del presente trabajo de investigación es tratar de profundizar en el estudio de la restauración arquitectónica en Galicia entre 1980 y 1990 , acercamiento que se realiza desde la disciplina de la historia del arte.

A finales de los años 70, España comenzaba a despertar de un letargo de cuarenta años que mantuvo al país aislado de las corrientes culturales europeas. Tras la aprobación de la Constitución de 1978, se inicia un camino hacia la recuperación, que en Galicia, al igual que en el resto del país, no finaliza hasta diez años después. Aunque en los años ochenta se inicia el debate sobre la restauración, el clima reinante es de gran confusión debido, sobre todo, a que los últimos tiempos habían estado protagonizados por esfuerzos colectivos más inmediatos y generales, marcados por una etapa de transición política y, a consecuencia de ello, lejanos, tanto de la especificidad que requiere este tipo de teorización como de una actitud comprometida frente a la conservación del patrimonio histórico. Se trata de analizar, pues, un proceso que se inicia y se desarrolla durante la década de los ochenta, se materializa en la formación de una actitud diferente con respecto a la protección, conservación y restaura-

"CUADERNOS DE ESTUDIOS GALLEGOS", Tomo XLIV, Fascículo 109, Santiago 1997. 
ción del patrimonio cultural, se consolida al inicio de la nueva década y se culmina, años más tarde, con la aprobación y publicación de la Ley do Patrimonio Cultural de Galicia.

El trabajo se inicia con la elaboración de un inventario de las actuaciones llevadas a cabo en toda Galicia. En una segunda fase, ya de catalogación, se centra el campo de investigación a la provincia de A Coruña. Estos proyectos arquitectónicos se estudian, más tarde, teniendo en cuenta el triple marco teórico, institucional y legislativo en el que se insertan. Es necesario mencionar que tanto en el apartado teórico, como en las diferentes normativas o recomendaciones internacionales se prioriza la búsqueda de la función que deben desempeñar los profesionales de la historia en la restauración arquitectónica y, debido a ello, los distintos temas y proyectos abordados, se valoran — fundamentalmente- atendiendo a esta perspectiva seleccionada.

\section{METODOLOGÍA DE TRABAJO}

\section{Inventario}

La investigación se inicia con la recopilación de las obras realizadas en Galicia durante la década de los ochenta. En esta labor de inventario se pueden establecer dos etapas claramente diferenciadas. La primera, comprendida entre 1980 y 1983; la segunda, entre 1984 y 1990. Esta división se debe a que los proyectos realizados en cada una de ellas están promovidos por distintos organismos y plantean problemáticas específicas, tanto a la hora de la recopilación de datos como en lo que al estudio estricto de las obras se refiere.

\section{Primera etapa: 1980 a 1983}

Fuentes.- Las fuentes documentales correspondientes a la primera etapa se consultaron en el Instituto de Conservación y Restauración de Bienes Culturales en Madrid, ya que en este organismo se conservan las referencias a las restauraciones promovidas por el Ministerio de Cultura clasificadas por Comunidades Autónomas. Sin embargo, la recopilación de los datos contenidos en los expedientes de las obras, memorias y planos, imprescindibles para la elaboración del catálogo, se localizaron entre los fondos de los archivos generales de este Ministerio. 
Marco institucional.- El inicio de este período coincide con un importante cambio en cuanto a la política de restauraciones se refiere: en el año 1980 tiene lugar la creación de un órgano administrativo específico dependiente del Ministerio de Cultura, la Secretaría General de Restauración, dirigida por el arquitecto Dionisio Hernández Gil, y un Servicio Técnico del que forman parte los arquitectos Manuel de las Casas y Antón Capitel, vinculados a las Cátedras de Proyectos y Composición Arquitectónicas de la Escuela Técnica Superior de Arquitectura de Madrid. Entre sus funciones figuraba la del traspaso de competencias a las distintas Comunidades Autónomas y la programación de encargos de los proyectos de restauración, hasta entonces misión del Consejo Asesor de Monumentos de la Dirección General de Bellas Artes.

Con la actividad desarrollada por este organismo especializado, se empieza a vislumbrar un cambio de actitud en cuanto a la política estatal de restauraciones se refiere. En el Ministerio de Cultura se trata de implicar en la restauración a todo el conjunto de la profesión arquitectónica que, hasta entonces, eran ajenos a los problemas de intervención en los edificios históricos. De esta forma se pasó, en tan sólo cinco años, de los treinta arquitectos que tradicionalmente trabajaban con la Dirección General de Bellas Artes a contar con cuatrocientos ochenta colaboradores. No obstante, esta decisión conlleva ciertos problemas, entre los que cabe destacar el entendimiento de la restauración no como un proyecto que precise una especialización, sino como un problema puro y exclusivamente arquitectónico ${ }^{1}$. Consideran que la restauración es una operación dedicada a la conservación, aplicando los escasos recursos disponibles de una manera extensiva, con objeto de poder alcanzar al mayor número de monumentos posible, por ello, la mayor parte de las actuaciones realizadas en esta etapa son consolidaciones estructurales, reparaciones de cubiertas, etc. Además, esta política prioriza la puesta en uso del mayor número de monumentos, lo que exige profundas operaciones de remodelación y ampliación, con objeto de adecuar los edificios a nuevos usos y funciones.

Paralelamente, la actitud de las nuevas instituciones democráticas, creadas en estos años de descentralización administrativa, hicieron que

\footnotetext{
' Humanes Bustamante, A.: «Introducción» en Intervenciones en el Patrimonio Arquitectónico 1980-1985. Ministerio de Cultura. I.R.C.B.C., Madrid, 1990, pp. 15-18.
}

"CUADERNOS DE ESTUDIOS GALLEGOS", Tomo XLIV, Fascículo 109, Santiago 1997. 
los monumentos pasaran del olvido casi absoluto a un protagonismo desmesurado, creando grandes expectativas a un colectivo profesional sin los recursos necesarios para hacer frente al reto que suponía la restauración monumental con la eficacia y corrección que hubieran sido deseables. El objetivo prioritario era, también, dotar a edificios civiles y religiosos de instalaciones y equipamientos adecuados, con el fin de albergar en ellos gran parte de las nuevas instituciones y servicios de las administraciones central, autónoma y municipal. Como consecuencia, se realizaron —en muchas ocasiones-drásticas intervenciones que modificaron considerablemente los monumentos.

Este tema había suscitado ya un interesante debate en el seno del Consejo de Europa del año 1975 así, en la Resolución número 'veintiocho de la Carta Europea del Patrimonio Arquitectónico, se proponía la terminología de «conservación integrada» para hacer referencia a la recomendable acción conjunta de las técnicas de la restauración y la búsqueda de funciones apropiadas para los edificios históricos. Frecuentemente, me he planteado, al llegar a este punto del discurso, que es imprescindible programar los nuevos usos en consonancia con las instalaciones existentes ya que nunca es aconsejable adoptar soluciones desacordes con las funciones para las que el edificio ha sido concebido, y así evitar con ello transgresiones en las fábricas. La adecuación entre forma y función, usos y usuarios es un tema que, a simple vista, parece evidente pero que se obvia la mayoría de las veces. No obstante, las causas de muchas actuaciones inadecuadas no siempre deben buscarse en un mal proyecto de restauración o reutilización, en las desacertadas soluciones constructivas de tal o cual arquitecto, sino en un mal encargo, circunstancia que condiciona la mayoría de las duras intervenciones que ha sufrido nuestro patrimonio arquitectónico.

Marco legal.- En estos primeros años de la década de los ochenta, estaba todavía en vigor la Ley del Patrimonio Artístico Nacional del 13 de mayo de 1933. Pocas veces, a lo largo de la historia de nuestro país, la legislación iba a situarse tan cerca de las corrientes teóricas más innovadoras existentes en materia de restauración. Pero, a pesar de ello, esta avanzada legislación quedó, en poco tiempo, convertida en mera utopía, ya que en las intervenciones que se aprueban y realizan bajo la supervisión de la Comisaría General del Patrimonio Artístico Nacional, orga-

"CUADERNOS DE ESTUDIOS GALLEGOS", Tomo XLIV, Fascículo 109, Santiago 1997. 
nismo que sustituye a la Junta Superior del Tesoro Artístico tras la guerra civil, se incumpliría totalmente. Se seguían únicamente los criterios de la más pura «reconstrucción en estilo», aunque sin la carga intelectual que esta teoría contenía. Por todo ello, a pesar de ser la única normativa sobre protección del patrimonio durante más de medio siglo, y del carácter innovador y progresista que la había inspirado, este cuerpo legal que, con todas sus limitaciones, había resultado adecuado para su época, ha sido incapaz de obtener resultados positivos en la protección del patrimonio debido, entre otras causas, a la no actualización de sus disposiciones que resultaron, con el tiempo, anacrónicas e insuficientes.

\section{Segunda etapa: 1984 a 1990}

Fuentes.- La documentación consultada para esta segunda etapa procede, primordialmente, del archivo de la Dirección Xeral do Patrimonio Histórico e Documental de la Consellería de Cultura. Si bien estas fuentes documentales presentaron menos dificultades de acceso que las referidas en la etapa anterior, la ausencia de sistematización o clasificación de los archivos y la carencia de una recopilación de las obras, promovidas por esta entidad, exigió - en primer lugar - una laboriosa tarea de inventariado así como la posterior búsqueda y localización de los expedientes.

Marco institucional.- Mediante el Real Decreto 2.434/1982, del 24 de julio, se traspasaron funciones y servicios de la Administración del Estado a la Comunidad Autónoma de Galicia en materia de cultura. Una vez transferidas las competencias, se crea la primera Consellería de Cultura, siendo su titular José Fernando Filgueira Valverde. Poco después, se especifica la estructura orgánica y funcional de la Consellería y, con ella, el organigrama de la primera Dirección Xeral do Patrimonio Artístico, Arqueolóxico e Histórico. Un año más tarde, la Consellería de Cultura pasa a denominarse Consellería de Educación y Cultura, y la Dirección Xeral a ostentar el rango administrativo de Subdirección Xeral dentro de la Dirección Xeral de Cultura; hasta que en diciembre de 1986 vuelve a recuperar la categoría de Dirección Xeral de Cultura e do Patrimonio Histórico-Artístico, de la Consellería de Cultura e Benestar Social. Por último, en el año 1988, se vuelve a modificar el organigrama adoptando la denominación de Consellería de Cultura e Deportes, en la que la Direc- 
ción Xeral adopta la designación de Dirección Xeral do Patrimonio Histórico e Documental.

Todos los cambios de estructura, anteriormente aludidos, no son más que un síntoma de la desorientación y desestabilización interna; prueba evidente de la falta de una línea de actuación coherente en cuanto a política cultural se refiere.

Marco legal.- Con la Ley de 1933 todavía en vigor y, por tanto, en un marco de obsolescencia legislativa, se va a producir el traspaso a Galicia de las competencias en materia de patrimonio, situación que se agrava considerablemente por la carencia de documentación: las nuevas competencias y la responsabilidad de la gestión no se ven acompañadas de los expedientes administrativos anteriores a las mismas. Esta documentación - proyectos, fotos y planos — siguen formando parte, todavía actualmente, de los fondos del archivo del Ministerio de Cultura.

El 25 de junio de 1985 se publica La Ley 16/85 del Patrimonio Histórico Español. Es importante destacar el cambio de filosofía que en ella se manifiesta, tanto en la propia definición de patrimonio, como en el tema de la tutela del patrimonio artístico se refiere; siguiendo las recomendaciones internacionales que establecían una doctrina diferente basada -incluso- en la formulación de un nuevo lenguaje centrado, a partir de los años setenta, en los Bienes Culturales y Ambientales. Pero su naturaleza eminentemente ecléctica, el intento de aglutinar la dispersión legislativa existente, muchas veces hasta de modo exhaustivo, hace que se configure como un conjunto de normas, a menudo inconexas e incluso - a veces-contradictorias.

La aplicación de esta normativa, válida en principio y con ciertos avances, se ha convertido con el tiempo en un instrumento ineficaz, puesto que no se articulan medidas paralelas de fomento de la cultura y tampoco se complementan las distintas disposiciones con adecuadas bonificaciones fiscales; las previstas, resultan inadecuadas e insuficientes para la realidad de un patrimonio cultural que precisa, urgentemente, tanto de las aportaciones de empresas privadas como de particulares, con objeto de garantizar su conservación. El Estado no es capaz de asumir y resolver las necesidades planteadas por un patrimonio tan rico como el que posee España en general y la Comunidad Autónoma de Galicia en particular. Es necesario potenciar una política fiscal que estimule las aportaciones de 
particulares por medio de mecenazgo, aspecto que está muy poco desarrollado en España, con respecto al resto de los países europeos o americanos.

Actuaciones. - Es interesante destacar la convocatoria, realizada a principios de los años ochenta, de los premios nacionales de restauración y rehabilitación de edificios monumentales, por la entonces Dirección General del Patrimonio Artístico, que serán concedidos a proyectos diseñados bajo diferentes criterios y planteamientos teóricos; hecho que supone la admisión de todo tipo de actuaciones en el patrimonio monumental por parte de la administración, hecho sin precedentes hasta entonces. Esta pluridad de criterios marca toda la década y el principio del fin de una larga etapa en la que el Estado encargaba sólo intervenciones muy específicas, centradas todas ellas en el historicismo y cerradas, casi siempre, a otra clase de cuestionamientos teóricos.

En Galicia también esta tendencia hacia la pluralidad de criterios en las actuaciones estará presente durante toda la década. Si bien, en principio, parece una postura adecuada y que pone de manifiesto las diferentes corrientes teóricas existentes, síntoma de la nueva preocupación que la restauración arquitectónica estaba despertando en los profesionales, induce a pensar que más que una preocupación se ha convertido en desorientación, por ser una línea de trabajo excesivamente sostenida. Se aprecia además, en todo el período estudiado, un importante desequilibrio entre las realizaciones prácticas y los debates teóricos. A pesar de que en los diez años estudiados se han realizado numerosas actuaciones sobre la arquitectura construida y que la restauración arquitectónica ha pasado a tener un papel importante en la actividad profesional de los arquitectos gallegos, las aportaciones teóricas y las publicaciones de los logros obtenidos no han complementado a la praxis.

Efectivamente, tampoco en Galicia, no puede hablarse de una política general en cuanto a las actuaciones en el patrimonio se refiere, ni se ha abordado el estudio del patrimonio cultural heredado de un modo global. Se han sufrido todos los inconvenientes de la administración joven, lejana del sedimento metodológico que sólo aporta la experiencia, únicamente conseguido tras largos años de actuaciones.

Pero es una evidencia contundente que a pesar de la enorme desproporción existente entre la cantidad de Bienes de Interés Cultural que po-

"CUADERNOS DE ESTUDIOS GALLEGOS", Tomo XLIV, Fascículo 109, Santiago 1997. 
see Galicia y los escasos recursos económicos destinados para su conservación, restauración o rehabilitación, y repasando las actuaciones de los últimos años, se puede hacer referencia a un gran número de proyectos coherentemente planteados y con soluciones constructivas acertadas, tanto por la teoría aplicada como por la praxis utilizada. Todo ello gracias a una espléndida generación de arquitectos que vinculados, en mayor o menor medida, a la Escuela Técnica Superior de Arquitectura de A Coruña pusieron todo su conocimiento profesional al servicio y la búsqueda de las soluciones proyectuales más adecuadas a cada caso concreto. Son proyectos que si bien no cambian la tónica general en un análisis de conjunto, en su mayoría obras de consolidación, restitución de cubiertas, drenajes perimetrales, etc., son muestras evidentes de que el debate de Galicia se había iniciado ya a principios de los años ochenta y que se desarrolla progresivamente a lo largo de toda la década.

\section{CATÁlogo}

Tras esta primera labor de inventariado, y debido a la ingente cantidad de información recopilada, se decide acotar el campo de estudio restringiéndolo únicamente a la provincia de A Coruña. Se da prioridad con esta elección al objetivo principal que guía este estudio: poder llegar a conocer en profundidad la elaboración de los proyectos, los estudios previos y la evolución de las obras realizadas, así como su resultado final, con el fin de extraer conclusiones en cuanto al papel desempeñado por el historiador del arte y la utilidad de su trabajo ante una actuación arquitectónica concreta. El fin último, es elaborar una propuesta metodológica idónea para la colaboración interdisciplinar en un proyecto de restauración en un Bien de Interés Cultural. Dado que la Ley de Patrimonio 16/85 establece que debe realizarse un estudio previo a las intervenciones en el patrimonio histórico construido, se programó este apartado - fundamentalmente- en base a los datos recogidos en las memorias de los proyectos, pensando utilizarlas como fuente principal de este estudio. Esto no ha sido posible, ya que una vez recopilada la información, se ha podido constatar que la mayor parte de las memorias de los proyectos de restauración arquitectónica no recogen ni tan siquiera los datos mínimos necesarios para una primera comprensión del inmueble.

"CUADERNOS DE ESTUdIOS GALLEGOS", Tomo XLIV, Fascículo 109, Santiago 1997. 
Al no ajustarse los supuestos de partida con la realidad encontrada, se decide el inicio de una investigación fundamentalmente bibliográfica y, en los casos en los que las lagunas encontradas lo hacía imprescindible, documental. Este apartado ha sido de vital importancia para adquirir la experiencia necesaria que ha posibilitado la elaboración de una propuesta metodológica, en cuanto al estudio histórico previo a la restauración arquitectónica se refiere, así como al modo de participación idóneo del historiador del arte, en la elaboración del proyecto de restauración, seguimiento de las obras e informe final de las mismas.

El nivel de conocimiento del edificio, por parte del técnico encargado de la redacción del proyecto o de la dirección de las obras; la evidencia de que éste se enfrenta de un modo consciente ante un monumento-documento sigue siendo una incógnita difícil de despejar, a la vista de la mayoría de las memorias de los proyectos de restauración analizados.

\section{Pero, ¿qué se entiende por monumento-documento?}

Tanto el origen del concepto monumento-documento como la noción actual de la historia del arte de la antigüedad nacen como consecuencia del contacto de la historia con la arqueología, concretamente, a partir de las excavaciones de Pompeya y Herculano ya que es precisamente a partir del estudio del primer catálogo de la colección real, publicado en 1755 tras los nuevos hallazgos obtenidos bajo el patrocinio de Carlos III, cuando el erudito alemán Johann Joachim Winckelmann presenta una relación sistematizada y ordenada cronológicamente de todo el arte antiguo.

El estudio de estos hallazgos arqueológicos desencadena toda una investigación histórica que tiene como consecuencia inmediata el nacimiento de la conciencia histórica. A partir de entonces, los monumentos son explicados como documentos de civilización y de cultura y las obras de arte ya no serán tomadas, en el futuro, como ideales a reproducir o emular, sino como manifestación artística y cultural del pasado, como testimonio de una época y por tanto, con valores definitivos y concluidos.

Surge así, paralelamente, el deseo de su transmisión —en el mejor estado de conservación posible- a las generaciones venideras, y de ello la necesidad de su restauración, considerada como un complejo número de operaciones destinadas no a actualizar el monumento, ni siquiera a enriquecerlo, sino a conservalo como testimonio de ese reconocido pasado.

"CUADERNOS DE ESTUDIOS GALLEGOS", Tomo XLIV, Fascículo 109, Santiago 1997. 
Aloïs Riegl, a principios del presente siglo, en su obra El culto moderno a los monumentos planteaba que el monumento debe ser considerado como un eslabón que forma parte de una larga cadena evolutiva, dada su condición de documento histórico. Pero es, una vez superada la valoración del edificio desde un punto meramente estilístico defendida por Viollet le Duc y del mero reconocimiento de su valor romántico e histórico preconizada por Ruskin, el momento en el que los arquitectos sienten la necesidad de afianzarse en la historia como fuente imprescindible para legitimar las diferentes fases constructivas del monumento y para restaurar el documento, del modo más científico posible, de acuerdo con las formas preexistentes. Esta tendencia, iniciada a finales del siglo XIX, no ha dejado de evolucionar requiriendo, cada vez de un modo más sistemático, de la colaboración de los profesionales de la historia en la elaboración de los estudios previos a los proyectos de restauración, así como de su asesoramiento técnico en el transcurso de las obras.

Un importante avance en este discurso se produce cuando Camilo Boito manifiesta que el monumento es una obra dual, arquitectónica e histórica a la vez, debiendo por ello ser respetados - según su teoría del restauro moderno - ambos valores. En la estimación del monumento como objeto arquitectónico, se debe tener en cuenta que las formas están condicionadas por una determinada función y que han sido concebidas, plásticamente, según los criterios estéticos imperantes en el momento de su construcción. Pero, dada la dualidad planteada anteriormente, hay que considerar no sólo la necesidad de conservar y transmitir sus valores puramente arquitectónicos, definidos tanto desde el punto de vista formal, de significado o simbología, sino también el propio valor histórico del monumento.

Giovannoni plantea que la arquitectura es el signo más auténtico de la historia que él mismo define como la permanencia de los símbolos de la civilización traducidos en piedra. Los acontecimientos pueden ser interpretados a través de los textos, mientras que las manifestaciones estéticas se expresan de un modo más objetivado.

Si se analizan correctamente estas dos premisas: la histórica y la estética, podemos deducir que los histórico no se circunscribe al momento concreto de la configuración del edifico sino que incluye toda manifestación constructiva posterior, toda huella del transcurso del tiempo ya se

"CUADERNOS DE ESTUDIOS GALLEGOS", Tomo XLIV, Fascículo 109, Santiago 1997. 
transcriba como añadidos, reforma, cambio de uso o simple pátina ${ }^{2}$. El monumento, así entendido, es una obra viva, cambiante, utilizada de diferentes maneras, según las distintas necesidades y funciones que le han sido adjudicadas; éstas han modificado, a lo largo del tiempo, tanto su concepción espacial como su disposición formal.

Pero es a partir del restauro crítico, inspirado en la obra de Benedetto Croce y proclamado por Roberto Pane y Cesare Brandi, cuando el proyecto de restauración se incorpora en la evolución histórico-artística del edificio, valorándolo como un acto creativo en sí mismo, cuestión que hará variar los planteamientos anteriores. Al considerar la restauración como una nueva aportación artística a la fábrica se admite que, con ella, se está incorporando al monumento un componente de la cultura arquitectónica moderna. Por otro lado, la nueva valoración estética de la obra arquitectónica, que implica un estudio individualizado del monumento, lleva al arquitecto a definirse por la aplicación de criterios particularizados a cada caso concreto y que lo conducen irrremediablemente al nométodo. Este es un interesante debate que debe suscitar la permanente reflexión de los profesionales de la historia sobre la valoración del pasado, al igual que la interpretación de la pretendida búsqueda de la «memoria colectiva», reflejo de una perfecta interacción entre pasado y presente.

Giovanni Carbonara profundiza aún más en la idea de que la consideración de la obra de arte no debe retrotraerse al momento de su concepción originaria, al momento histórico en el que se crea, sino que es imprescindible reconocer como tal todos los elementos que se le han ido incorporando a lo largo de los años. Así, esta doble valoración, histórica y estética, confluyen en la necesidad de convertir a la restauración en un acto crítico ya que el arquitecto tiene la posiblidad de establecer un juicio crítico, histórico y estético de la obra de arte sobre la que ha de intervenir, de ahí la necesidad de establecer el inicio de un profundo estudio filológico del monumento.

Si partíamos de la base de que un objeto arquitectónico tiene entre sus dos básicas características la de ser documento de la historia, ¿quién mejor que un historiador, especialista en descifrar y valorar los registros del

\footnotetext{
${ }^{2}$ Mora Alonso Muñoyerro, S.: «La restauración monumental en España (1850-1939) en Quaderns Cientifics y Tècnics. Diputaciò de Barcelona. Servei del Patrimoni Arquitectònic Local. Barcelona 1993, pp. 11-18.
}

"CUADERNOS DE ESTUDIOS GALLEGOS", Tomo XLIV, Fascículo 109, Santiago 1997. 
pasado, para entender la evolución que el monumento ha seguido a lo largo de los siglos, para establecer el reivindicado estudio filológico del monumento?. Su aportación al estudio previo a toda restauración, legitimada ya como parte de esa larga cadena evolutiva, es no sólo incuestionable sino imprescindible.

\section{El proyecto como documento del «libro de fábrica» del monumento}

Viollet le Duc ya planteaba, como parte esencial de su metodología de restauración en estilo, la necesidad de una investigación previa a toda intervención, y puntualizaba que ésta tenía que ser exhaustiva, documental e histórica, con objeto de profundizar en el complejo conocimiento del monumento. Establecía también que el estudio físico-constructivo del edificio, basado en el análisis racional de su estructura era una premisa indispensable a la hora de abordar cualquier actuación. Esta investigación documental e histórica, la completaba con levantamientos gráficos y fotográficos, considerando a todos ellos medios imprescindibles para elaborar una metodología de trabajo coherente ${ }^{3}$.

La Carta de Atenas (1931), en su apartado VI, reconoce al monumento como documento de civilización y cultura, pero a pesar de ello no especifica ningún tipo de recomendación con relación a la documentación previa a la intervención, únicamente determina que las supresiones de la fábrica deben quedar debidamente documentadas y expuestas en un lugar próximo del monumento.

Sin embargo, la primera Carta del Restauro italiana, también de 1931, le dedica una especial atención al tema, planteando que: "Como en las excavaciones, así también en la restauración de los monumentos será condición esencial y determinante que una documentación precisa acompañe a los trabajos además de una relación analítica recogida en un diario de restauración e ilustrada con dibujos y fotografias, de modo que todos los elementos determinados en la estructura y en la forma del monumento, todas las fases de la obra de recomposición, de liberación, de complementación, queden reflejadas de modo permanente y seguro» ${ }^{4}$.

${ }^{3}$ Viollet le Duc, E.: Dictionnaire raisonné de l'architteture française du XI au XVI siècle. B. Bauce-A. Morel, París 1854-68.

${ }^{4}$ Ceschi, Carlo: Teoria e storia del restauro. Mario Bulzoni Editori. Roma 1970.

"CUADERNOS DE ESTUDIOS GALLEGOS", Tomo XLIV, Fascículo 109, Santiago 1997. 
Pero el progreso más importante se produce con la publicación de la Carta de Venecia de 1964 ya que, por primera vez, se admite en un documento de ámbito internacional que «La conservación y restauración de los monumentos constituye una disciplina que reclama la colaboración con todas las ciencias y con todas las técnicas que pueden contribuir al estudio y a la protección del patrimonio monumental"5; especificando en su artículo noveno que «La restauración estará siempre precedida y acompañada por un estudio arqueológico e histórico del monumento ${ }^{6}$.

En la Carta del Restauro de 1972, inspirada en la teoría de Cesare Brandi, de nuevo se reclama que cada intervención debe ser estudiada previamente y argumentada por escrito. Durante el desarrollo de las obras se aconseja también la realización de un diario de operaciones efectuadas, al que seguirán una relación final, con la documentación gráfica de antes, durante, y después de la intervención. Se intensifica, cada vez más, no sólo la necesidad del estudio previo a la actuación, sino la documentación del seguimiento de las obras y el informe final de las mismas.

En relación con este tema de la documentación histórica, la última Carta del Restauro de 1987 es más drástica todavía, estableciendo entre sus conclusiones que ningún proyecto de conservación o restauración podrá considerarse idóneo para pasar a la fase de ejecución si no está precedido, de un estudio atento de la obra y de su contexto ambiental. Parte integrante de este estudio serán las investigaciones bibliográficas, iconográficas y de archivos, con objeto de adquirir todos los datos históricos posibles, además de investigaciones experimentales sobre las propiedades materiales del inmueble. Será necesario en tal fase atribuir la máxima importancia a la historia de las transformaciones materiales del monumento recabando información, en especial, en relación a sus diferentes reutilizaciones?

\section{Los estudios previos en los proyectos de restauración}

A lo largo de esta investigación se ha podido constatar como la mayor parte de las memorias de los proyectos de restauración arquitectónica no recogen ni tan siquiera los datos mínimos necesarios para una primera

\footnotetext{
${ }^{5}$ Carta de Venecia, mayo 1964: artículo 1.

${ }^{6}$ Idem, artículo 9.

${ }^{7}$ Martínez Justicia, M.J.: op. cit., p. 41.
}

"CUADERNOS DE ESTUDIOS GALLEGOS", Tomo XLIV, Fascículo 109, Santiago 1997. 
comprensión del edificio. Dichos estudios son imprescindibles, a mi entender, para que el arquitecto haga una correcta interpretación de la fábrica ya que sólo profundizando en el conocimiento de su realidad arquitectónica podrá llegar a deducir la diagnosis que solucione las patologías que presente el edificio y así, mediante la utilización de las soluciones constructivas más adecuadas, y aplicando los criterios de restauración con el rigor científico que requiere una actuación en un Bien de Interés Cultural pueda redactar primero, y dirigir después, un adecuado proyecto de restauración.

Todavía en la actualidad este aspecto de la restauración arquitectónica es frecuentemente olvidado por los arquitectos debido, probablemente, a que la mayoría de ellos consideran que la memoria histórica es un trámite más a cubrir en la presentación oficial de un proyecto de restauración, quizás, desconociendo la rentabilidad proyectual que les puede proporcionar una adecuada lectura científica del monumento.

En Galicia, salvo casos aislados, los proyectos de restauración no cuentan con memoria histórica y en los proyectos a los que se acompaña una reseña, la mayor parte de las veces, no está realizada por los técnicos adecuados; frecuentemente, son los mismos arquitectos los que, de modo particular, inician una investigación para la que no están suficientemente cualificados.

Sin embargo, la mayoría de los proyectos consultados para este trabajo, contienen una útil documentación gráfica, a pesar de que frecuentemente no se completa con planos de las variaciones derivadas de la actuación prevista inicialmente. La documentación fotográfica, por el contrario, es prácticamente inexistente, pese a que un reportaje fotográfico de la situación en la que se encontraba el edificio antes de la intervención, del avance de las obras y de las soluciones finalmente adoptadas, son documentos que no sólo están previstos en todas las normativas y recomendaciones internacionales, sino que constituyen fuentes documentales de vital importancia para poder afrontar, en el futuro, las contingencias que pueda sufrir el monumento.

\section{La actuación arquitectónica y el seguimiento de las obras}

El seguimiento de las obras y las transformaciones o alteraciones que se producen a lo largo de las mismas, en muy raras ocasiones están documentadas. Por ello, a menudo, cuando se realiza el trabajo de campo con

"CUADERNOS DE ESTUDIOS GALLEGOS", Tomo XLIV, Fascículo 109, Santiago 1997. 
objeto de iniciar el estudio físico-constructivo del edificio y comprobar los resultados de la restauración o restauraciones arquitectónicas anteriores, se ha podido constatar que, en muy pocas ocasiones, los proyectos coinciden con la realidad. Se entiende que esta circunstancia no sólo es frecuente sino que es - en cierto modo- lógica debido a que la práctica de la restauración monumental exige la dedicación, paciencia y pericia artesanal de todo el equipo técnico que la lleva a cabo y que, a lo largo de la evolución de los trabajos, los proyectos pueden ser modificados e incluso profundamente alterados puesto que es prácticamente imposible prever cómo se encuentra realmente el estado de la fábrica en una primero observación del monumento. Por ello, a menudo, los proyectos se convierten - únicamente- en un avance de intenciones, que poco o nada tienen que ver con su resultado final.

Hasta este punto de la reflexión me identifico plenamente con el director de las obras, pero lo que me resulta totalmente incomprensible es que, si realmente todos los profesionales que actúan en el patrimonio construido tienen consciencia de que el monumento es un documento, no se tomen la cautela de reflejar estas modificaciones en los informes mensuales de la obra y, más tarde, en el informe final de las mismas puesto que serán parte importantísima del «libro de fábrica» del inmueble.

\section{Reflexiones desde la historia del arte}

Como resultado de esta investigación se plantea una propuesta metodológica. Una reflexión acerca del método capaz de adaptar la investigación histórica tradicional a los problemas derivados de las necesidades específicas del proyecto. Se establece, «a priori», que la obra arquitectónica no sólo está concebida según unos criterios estéticos determinados, sino que también ha recibido la impronta de numerosas etapas constructivas, y con ellas, la huella de su historia, de ahí proviene el indiscutible interés que tiene su estudio filológico. Además del análisis arquitectónico, se plantea la necesidad de tener en cuenta otros factores que contribuyen a definir y configurar el objeto como son: la escultura, la pintura, la iluminación, la distribución espacial o las funciones y usos a los que el monumento ha estado dedicado, así como distintos condicionantes derivados de ellos.

Es conveniente también iniciarse en el conocimiento de campos de trabajo que puedan resultar complementarios para el estudio físico-constructivo de los monumentos, como es el espacio de la lectura estratigráfica 
de paramentos, utilizada en los últimos años por distintos arqueólogos que siguiendo las investigaciones de Harris ha sido desarrollada ampliamente por los arquitectos Brogiolo y Parenti de la Universidad de Siena.

La admisión de que el monumento es un documento supone que cualquier intervención en un edificio, considerado portador de una rica información estratificada en sus fábricas, ha de afrontarse con el mayor grado de conocimiento y respeto posibles. Para ello, es necesario plantearse la revisión de las disciplinas e iniciar una profunda reflexión por parte de todos los profesionales que, de un modo u otro, intervienen en el proceso de restauración monumental: arqueólogos, historiadores, historiadores del arte, químicos restauradores, arquitectos, con objeto de tratar de adaptar una metodología de trabajo individual, a una colaboración interdisciplinar.

La revisión de la historia del arte debe conducir a la búsqueda de la metodología que resulte más adecuada para conseguir una correcta lectura científica del monumento, huyendo de los trabajos excesivamente amplios y poco prácticos para ser utilizados por un técnico que necesita respuestas concretas para preguntas concretas. Las generalizaciones excesivas y las profundas adscripciones a corrientes estilísticas o a períodos históricos muy precisos no pueden excluir lo fundamental a la hora de realizar un informe previa a la actuación en un Bien de Interés Cultural. El análisis de las fábricas y el estudio directo del monumento debe ser siempre prioritario ya que, como se ha expuesto repetidamente, éste es considerado como documento de civilización y cultura. El historiador del arte utilizar en su investigación al monumento como fuente documental básica para su estudio. La eliminación, por falta de comprensión, de algún elemento del monumento puede dejar «muda» para la historia una parte importante de la «memoria colectiva» que, plasmada en piedra, aporta no sólo datos histórico-constructivos, sino también referencias culturales y sentimentales del lugar en el que éste se emplaza.

En esta reflexión no se deben olvidar las restauraciones anteriores debido a que toda intervención posterior a la fábrica forma parte inseparable de la historia del edificio. Además, como ya se insinuaba anteriormente, el historiador ha de obviar posturas conservacionistas a las que tradicionalmente se adscribe, para aceptar y tener en cuenta que el arquitecto de cada época interviene en la fábrica desde su propia concepción de la arquitectura, con una metodología determinada y unos criterios de actuación concretos.

"CUADERNOS DE ESTUDIOS GALLEGOS", Tomo XLIV, Fascículo 109, Santiago 1997. 
Por ello creo que el no-método, la consciencia de que cada edificio es una individualidad que precisa de ser abordada sin ningún planteamiento hecho «a priori» para llegar a un profundo conocimiento científico del objeto sobre el que se ha de actuar es, posiblemente, la actitud más acertada.

$\mathrm{Si}$, según se ha ido exponiendo a lo largo de estas páginas, los ciudadanos del siglo veinte hemos aprendido a valorar las aportaciones de todas las épocas al edificio. ¿Por qué entonces negar la contribución estética de la restauración actual?. 